Теорія Ймовір. та Матем. Статист. Вип. 76, 2007
Theor. Probability and Math. Statist.

No. 76, 2008, Pages 103-116

S 0094-9000(08)00735-7

Article electronically published on July 14, 2008

\title{
A THEOREM ON THE DISTRIBUTION OF THE RANK OF A SPARSE BOOLEAN RANDOM MATRIX AND SOME APPLICATIONS
}

UDC 519.21

\author{
V. I. MASOL AND S. V. POPERESHNYAK
}

\begin{abstract}
We consider some estimates of the rate of convergence of the distribution of a sparse Boolean random matrix to the Poisson distribution. The results obtained in the paper are applied to estimate the probability that a nonhomogeneous system of Boolean random linear equations is consistent.
\end{abstract}

\section{INTRODUCTION}

Different methods are used in [1] and [2] to prove that the distribution of the $\operatorname{rank} r(A)$ of a sparse Boolean random matrix $A$ approaches the Poisson distribution as

$$
T=T(n) \rightarrow \infty \quad \text { and } n \rightarrow \infty
$$

(here $n$ and $T$ mean the number of columns and rows in the matrix $A$, respectively). If $T$ and $n$ are finite, then the distribution of the rank $r(A)$ can be expressed in terms of the factorial moments of the random variable $r(A)$. These expressions are found in [3]. The asymptotic behavior as $n \rightarrow \infty$ of the factorial moments is given in [4, Theorem 4] under certain assumptions, say if $n-T=$ const and if the distribution of entries of the matrix $A$ depends on the number of the column only.

We are interested in the bounds of the rate of convergence of the distribution of the rank of the matrix $A$ to the Poisson distribution as $n$ grows and in applications of these bounds for an estimation of the probability that the following nonhomogeneous system of linear equations is consistent:

$$
A X=B
$$

where the entries of the matrix $A$ of coefficients belong to the field $G F(2)$ consisting of two elements, the vector column $B=\left(b_{1}, \ldots, b_{T}\right)^{\prime}$ is formed by the random variables $b_{1}, \ldots, b_{T}$ that do not depend on the entries of the matrix $A$ and moreover $b_{1}, \ldots, b_{T}$ are jointly independent random variables assuming values in $G F(2)$ and having known distributions, and where $X=\left(x_{1}, x_{2}, \ldots, x_{n}\right)^{\prime}$ is an $n$-dimensional column vector such that $x_{i} \in G F(2), i=1, \ldots, n$.

In contrast to the papers [1, 2], we consider random matrices $A$ whose entries have the distributions that may depend on their position in the matrix $A$.

2000 Mathematics Subject Classification. Primary 68U20; Secondary 60G10.

Key words and phrases. Boolean random matrix, rank of a matrix, the probability that a system is consistent, the rate of convergence of distributions.

(C)2008 American Mathematical Society 


\section{MAIN RESUlTS}

Let the entries of a $T \times n$ matrix $A=\left\|a_{i j}\right\|, i=1, \ldots, T, j=1, \ldots, n$, be independent random variables assuming values in $G F(2)$ and whose distributions are given by

$$
\mathrm{P}\left\{a_{i j}=1\right\}=1-\mathrm{P}\left\{a_{i j}=0\right\}=\frac{\ln n+x_{i j}}{n},
$$

where

$$
\left|x_{i j}\right| \leq c, \quad c=\text { const }, i=1, \ldots, T, j=1, \ldots, n .
$$

We assume that the matrix $A$ has at least $n_{0}>1$ columns; thus the distributions (2) are well defined for $n \geq n_{0}$. Note that a matrix $A$ whose entries have the distributions given by (2) $-(3)$ is called a sparse Boolean matrix.

Denote by $r(A)$ the rank of the matrix $A$ and put

$$
\lambda=\frac{1}{n} \sum_{i=1}^{T} \exp \left\{-\frac{1}{n} \sum_{j=1}^{n} x_{i j}\right\} .
$$

Theorem 2.1. Let conditions (2) and (3) hold. If

$$
\frac{T}{n} \leq 1-\frac{\log _{2} \ln n}{(\ln n)^{q}}, \quad q=\mathrm{const}, 0<q<1,
$$

then

$$
\left|\mathrm{P}\{r(A)=T-k\}-\frac{e^{-\lambda} \cdot \lambda^{k}}{k !}\right| \leq 2(1+\delta) c(n, k) \frac{\ln ^{4} n}{n(\ln \ln n)^{2}}
$$

for $k=0,1,2, \ldots, n$.

If, additionally,

$$
\varliminf_{n \rightarrow \infty} \frac{T}{n}>0
$$

then

$$
1<\varliminf_{n \rightarrow \infty} c(n, k) \leq \varlimsup_{n \rightarrow \infty} c(n, k) \leq e^{e^{c}},
$$

where $\delta=$ const $>0$. The explicit expression for the coefficient $c(n, k)$ is given by equality (40).

Further we assume that

$$
\mathrm{P}\left\{a_{i j}=1\right\}=1-\mathrm{P}\left\{a_{i j}=0\right\}=\frac{\ln T+x_{i j}}{T}
$$

and that restrictions (3) are satisfied. Put

$$
\lambda_{1}=\frac{1}{T} \sum_{j=1}^{n} \exp \left\{-\frac{1}{T} \sum_{i=1}^{T} x_{i j}\right\} .
$$

Theorem 2.2. Let conditions (3) and (7) hold. If

$$
1+\frac{\log _{2} \ln T}{(\ln T)^{q}} \leq \frac{T}{n}, \quad q=\text { const }, 0<q<1,
$$

then

$$
\left|\mathrm{P}\{r(A)=n-k\}-\frac{e^{-\lambda_{1}} \cdot \lambda_{1}^{k}}{k !}\right| \leq 2(1+\delta) c(T, k) \frac{\ln ^{4} T}{T(\ln \ln T)^{2}}
$$

for $k=0,1,2, \ldots, T$ where $\delta=$ const $>0$. 
If, additionally,

$$
\varlimsup_{n \rightarrow \infty} \frac{T}{n}<\infty
$$

then

$$
1<\varliminf_{n \rightarrow \infty} c(T, k) \leq \varlimsup_{n \rightarrow \infty} c(T, k) \leq e^{e^{c}} .
$$

Consider system (11) where the entries of the $T \times n$ matrix $A=\left\|a_{i j}\right\|$ are independent random variables whose distributions satisfy conditions (2)-(3) for $i=1, \ldots, T$, $j=1, \ldots, n$.

The column vector $B=\left(b_{1}, \ldots, b_{T}\right)^{\prime}$ consists of random variables $b_{1}, \ldots, b_{T}$ that are jointly independent, do not depend on the entries of the matrix $A$, and assume values 0 and 1 with probabilities

$$
\mathrm{P}\left\{b_{i}=0\right\}=\frac{1}{2}\left(1+\varepsilon_{i}(n)\right), \quad i=1,2, \ldots, T .
$$

Denote by $P_{n, T}$ the probability that system (1) is consistent.

Theorem 2.3. Let

$$
\frac{T}{n} \leq 1-\frac{\log _{2} \ln n}{(\ln n)^{q}}, \quad q=\text { const }, 0<q<1,
$$

for all $n \geq n_{0}>1$. Assume that conditions (2), (3), and (8) hold. If

$$
\tilde{\varepsilon}(n) \leq \beta<1 \text { and } \beta=\text { const, }
$$

where

$$
\tilde{\varepsilon}(n)=\max _{1 \leq k \leq T}\left|\varepsilon_{k}(n)\right|,
$$

then

$$
-\alpha_{2} f(n)-\tilde{\varepsilon}(n) \leq P_{n, T}-\exp \left\{-\frac{\lambda}{2}\right\} \leq \alpha_{1} f(n)+\tilde{\varepsilon}(n) .
$$

Here

$f(n)=4(1+\delta) e^{e^{c}} \frac{\ln ^{4} n}{n(\ln \ln n)^{2}}, \quad \alpha_{1}=1+\frac{1}{1-\tilde{\varepsilon}(n)}, \quad \alpha_{2}=1+\Delta(n, T)+\frac{1}{1+\tilde{\varepsilon}(n)}$, $\delta=$ const $>0$, and

$$
0 \leq \Delta(n, T) \leq\left(\frac{\lambda e}{2 T}\right)^{T+1} \frac{\sqrt{2 T}}{2 T-\lambda e}(f(n))^{-1} .
$$

If, additionally, condition (6) holds, then

$$
\Delta(n, T) \rightarrow 0 \quad \text { as } n \rightarrow \infty .
$$

\section{Auxiliary Results for the Proof of Theorem 2.1}

Denote by $\xi_{n, T}$ the number of nonzero rows in the matrix $A$.

Lemma 3.1. If conditions (2) and (3) hold, then

$$
\left|\mathrm{P}\left\{\xi_{n, T}=k\right\}-\frac{e^{-\lambda} \cdot \lambda^{k}}{k !}\right| \leq Q(n, k)
$$

for $k=0,1,2, \ldots, n$, where

$$
Q(n, k)=\frac{\ln ^{2} n}{n} c_{1}(n, k)
$$

and the coefficient $c_{1}(n, k)$ is given by equality (18). 
If, additionally,

$$
\frac{T}{n} \leq 1
$$

then

for $k=0$, and

$$
0<\varliminf_{n \rightarrow \infty} c_{1}(n, k) \leq \varlimsup_{n \rightarrow \infty} c_{1}(n, k) \leq e^{-1}
$$

$$
0<\varliminf_{n \rightarrow \infty} c_{1}(n, k) \leq \varlimsup_{n \rightarrow \infty} c_{1}(n, k) \leq \frac{k^{k}}{k !} e^{-k} \max \left(k, e^{c}\right)
$$

for $k \geq 1$.

Proof. It is clear that the probability $p_{n}^{(i)}$ that a row $i$ of the matrix $A$ is constituted of zeros is equal to

$$
p_{n}^{(i)}=\prod_{j=1}^{n}\left(1-\frac{\ln n+x_{i j}}{n}\right), \quad i=1, \ldots, T .
$$

Put $a=p_{n}^{(1)}+p_{n}^{(2)}+\cdots+p_{n}^{(T)}$.

According to the Poisson theorem [5],

$$
\left|\mathrm{P}\left\{\xi_{n, T}=k\right\}-\frac{e^{-a} \cdot a^{k}}{k !}\right| \leq \frac{1}{n^{2}} \sum_{i=1}^{T} \exp \left\{-\frac{2}{n} \sum_{j=1}^{n} p_{i j}\right\} .
$$

Using (2) and (3) we find that

$$
\left|\frac{\lambda^{k} e^{-\lambda}}{k !}-\frac{a^{k} e^{-a}}{k !}\right| \leq \frac{\lambda^{k} e^{-\lambda} \gamma_{n}}{k !} \max \left\{k, \lambda\left(1+\frac{\lambda \gamma_{n}}{2} e^{\lambda \gamma_{n}}\right)\right\},
$$

where

$$
\gamma_{n}=\left(\frac{\ln ^{2} n}{2 n}\right) \frac{\left(1-c(\ln n)^{-1}\right)^{2}}{1-(\ln n-c) n^{-1}} .
$$

Combining (16) and (17) we see that

$$
\left|\mathrm{P}\left\{\xi_{n, T}=k\right\}-\frac{e^{-\lambda} \cdot \lambda^{k}}{k !}\right| \leq Q(n, k),
$$

where

and

$$
Q(n, k)=\frac{\ln ^{2} n}{n} c_{1}(n, k)
$$

$$
\begin{aligned}
c_{1}(n, k)= & \frac{1}{n \ln ^{2} n} \sum_{i=1}^{T} \exp \left\{-\frac{2}{n} \sum_{j=1}^{n} p_{i j}\right\} \\
& +\frac{\lambda^{k} e^{-\lambda}}{2 k !} \frac{\left(1-c(\ln n)^{-1}\right)^{2}}{1-(\ln n-c) n^{-1}} \max \left\{k, \lambda\left(1+\frac{\lambda \gamma_{n}}{2} e^{\lambda \gamma_{n}}\right)\right\} .
\end{aligned}
$$

One can use (2), (3), and (15) to estimate the coefficient $c_{1}(n, k)$; namely, for $k=0$,

$$
0<\varliminf_{n \rightarrow \infty} c_{1}(n, k) \leq \varlimsup_{n \rightarrow \infty} c_{1}(n, k) \leq e^{-1}
$$

and for $k \geq 1$,

$$
0<\varliminf_{n \rightarrow \infty} c_{1}(n, k) \leq \varlimsup_{n \rightarrow \infty} c_{1}(n, k) \leq \frac{k^{k}}{k !} e^{-k} \max \left(k, e^{c}\right) .
$$

Lemma 3.1 is proved. 
Denote by $S_{1}(A)$ the total number of independent critical sets of the matrix $A$ such that each of them has at least one nonzero row. (Recall [2] that a set $C=\left\{t_{1}, \ldots, t_{m}\right\}$, $m \in\{1,2, \ldots, T\}$, of indices of rows in a matrix $A$ is called a critical set if

$$
a_{t_{1}} \oplus \cdots \oplus a_{t_{m}}=0
$$

where the sum is understood coordinate-wise, $a_{t}=\left(a_{t 1}, \ldots, a_{t n}\right)$, and $\oplus$ is the symbol of addition in the field $G F(2)$. Critical sets $C_{1}, \ldots, C_{s}$ are called independent if the equality $\varepsilon_{1} C_{1} \Delta \varepsilon_{2} C_{2} \Delta \cdots \Delta \varepsilon_{s} C_{s}=0$ is equivalent to $\varepsilon_{1}=\varepsilon_{2}=\cdots=\varepsilon_{s}=0$, where $\varepsilon_{i} \in\{0,1\}$, $i, j \in\{1,2, \ldots, s\}$, and if

$$
C_{i} \Delta C_{j}=\left(C_{i} \cup C_{j}\right) \backslash\left(C_{i} \cap C_{j}\right)
$$

for different critical sets $C_{i}$ and $C_{j}$.

Lemma 3.2. If condition (2) holds, then the mathematical expectation of the random variable $S_{1}(A)$ is given by

$$
\begin{aligned}
\mathrm{E} S_{1}(A)= & \sum_{k=0}^{T} \sum_{1 \leq i_{1}<\cdots<i_{k} \leq T} \frac{1}{2^{n}} \prod_{j=1}^{n}\left(1+\prod_{s=1}^{k}\left(1-\frac{2\left(\ln n+x_{i_{s} j}\right)}{n}\right)\right) \\
& -\sum_{k=0}^{T} \sum_{1 \leq i_{1}<\cdots<i_{k} \leq T} \prod_{s=1}^{k} \prod_{j=1}^{n}\left(1-\frac{\ln n+x_{i_{s} j}}{n}\right) .
\end{aligned}
$$

Proof. Lemma 3.2 is proved similarly to the corresponding assertion in [2], where the expression for the mathematical expectation of $\mathrm{E} S_{1}(A)$ is obtained for the case of

$$
x_{i j}=x, \quad i=1, \ldots, T, j=1, \ldots, n .
$$

Put

$$
f(n)=\left[(\ln \ln n)^{-1}\left(1+\delta_{1}\right) \ln n\right]
$$

where $\delta_{1}=$ const, $\delta_{1}>0$, and

$$
\mu(n)=\sum_{k=0}^{f(n)} \sum_{1 \leq i_{1}<\cdots<i_{k} \leq T} \exp \left\{-\sum_{s=1}^{k} \sum_{j=1}^{n} \frac{\ln n+x_{i_{s} j}}{n}\right\} .
$$

Here $[\cdot]$ denotes the integer part.

Lemma 3.3. Assume that conditions (2), (3), and (6) are valid. If

$$
T \leq n,
$$

then

$$
1<\varliminf_{n \rightarrow \infty} \mu(n) \leq \varlimsup_{n \rightarrow \infty} \mu(n) \leq e^{e^{c}} .
$$

Proof. First we estimate $\mu(n)$ from above. The sum $\mu(n)$ can be represented in the following form:

$$
\mu(n)=\theta_{1}(n)-\theta_{2}(n),
$$

where

$$
\begin{gathered}
\theta_{1}(n)=\sum_{k=0}^{T} \sum_{1 \leq i_{1}<\cdots<i_{k} \leq T} \exp \left\{-\sum_{s=1}^{k} \sum_{j=1}^{n} \frac{\ln n+x_{i_{s} j}}{n}\right\}, \\
\theta_{2}(n)=\sum_{k=f(n)+1}^{T} \sum_{1 \leq i_{1}<\cdots<i_{k} \leq T} \exp \left\{-\sum_{s=1}^{k} \sum_{j=1}^{n} \frac{\ln n+x_{i_{s} j}}{n}\right\} .
\end{gathered}
$$


Then

$$
\theta_{1}(n) \leq e^{\lambda} .
$$

Since $\theta_{2}(n) \geq 0$, we derive from (21) and (22) that

$$
\mu(n) \leq e^{\lambda} .
$$

Now we estimate $\mu(n)$ from below. We have

$$
\theta_{1}(n) \geq e^{\lambda} \exp \left\{-\frac{1}{2 n^{2}} \sum_{i=1}^{T} \exp \left(-\frac{2}{n} \sum_{j=1}^{n} x_{i j}\right)\right\} .
$$

Taking into account representation (21) we obtain

$$
\mu(n) \geq e^{\lambda} \exp \left\{-\frac{1}{2 n^{2}} \sum_{i=1}^{T} \exp \left(-\frac{2}{n} \sum_{j=1}^{n} x_{i j}\right)\right\}-\theta_{2}(n) .
$$

The latter inequality together with (23) yields

$$
e^{\lambda} \exp \left\{-\frac{1}{2 n^{2}} \sum_{i=1}^{T} \exp \left(-\frac{2}{n} \sum_{j=1}^{n} x_{i j}\right)\right\}-\theta_{2}(n) \leq \mu(n) \leq e^{\lambda} .
$$

Thus

$$
\theta_{2}(n) \rightarrow 0 \quad \text { as } n \rightarrow \infty .
$$

Now we pass to the limit as $n \rightarrow \infty$ in inequality (24) and get (20) by relation (25) and conditions (3), (6), and (19).

Lemma 3.3 is proved.

Put

$$
\Gamma(n)=u\left(1+\frac{u\left(1+\delta_{1}\right)}{2} e^{u\left(1+\delta_{1}\right)}\right), \quad u=\frac{\ln ^{4} n}{n(\ln \ln n)^{2}} .
$$

Lemma 3.4. If conditions (2) and (3) are valid, then

$$
\begin{aligned}
& \sum_{k=0}^{f(n)} \sum_{1 \leq i_{1}<\cdots<i_{k} \leq T} \frac{1}{2^{n}} \prod_{j=1}^{n}\left(1+\prod_{s=1}^{k}\left(1-\frac{2\left(\ln n+x_{i_{s} j}\right)}{n}\right)\right) \\
& \leq \mu(n)+\mu(n)(1+\delta) \Gamma(n) .
\end{aligned}
$$

Proof. We estimate the left hand side of relation (26) as follows:

$$
\begin{aligned}
& \sum_{k=0}^{f(n)} \sum_{1 \leq i_{1}<\cdots<i_{k} \leq T} \frac{1}{2^{n}} \prod_{j=1}^{n}\left(1+\prod_{s=1}^{k}\left(1-\frac{2\left(\ln n+x_{i_{s} j}\right)}{n}\right)\right) \\
& \leq \sum_{k=0}^{f(n)} \sum_{1 \leq i_{1}<\cdots<i_{k} \leq T} \prod_{j=1}^{n}\left(1-\sum_{s=1}^{k} \frac{\left(\ln n+x_{i_{s} j}\right)}{n}+\left(\sum_{s=1}^{k} \frac{\left(\ln n+x_{i_{s} j}\right)}{n}\right)^{2}\right) .
\end{aligned}
$$

The latter inequality with the help of (3) implies that

$$
\begin{aligned}
& \sum_{k=0}^{f(n)} \sum_{1 \leq i_{1}<\cdots<i_{k} \leq T} \frac{1}{2^{n}} \prod_{j=1}^{n}\left(1+\prod_{s=1}^{k}\left(1-\frac{2\left(\ln n+x_{i_{s} j}\right)}{n}\right)\right) \\
& \leq \mu(n) \cdot \exp \left\{\frac{f^{2}(n)(\ln n+c)^{2}}{n}\right\} .
\end{aligned}
$$


Further

$$
\exp \left\{\frac{f^{2}(n)(\ln n+c)^{2}}{n}\right\} \leq 1+u(1+\delta)\left(1+\frac{u}{2}(1+\delta) \cdot \exp \{u(1+\delta)\}\right) .
$$

Relations (27) and (28) imply inequality (26).

Lemma 3.4 is proved.

Lemma 3.5. Let conditions (2), (3), (6), and (19) hold. Then

$$
\sum_{k=0}^{f(n)} \sum_{1 \leq i_{1}<\cdots<i_{k} \leq T} \prod_{s=1}^{k} \prod_{j=1}^{n}\left(1-\frac{\ln n+x_{i_{s} j}}{n}\right) \geq \mu(n)-c_{2}(n) \frac{\ln ^{3} n}{n \cdot \ln \ln n},
$$

where

$$
\frac{\left(1+\delta_{1}\right)}{2}<\varliminf_{n \rightarrow \infty} c_{2}(n) \leq \varlimsup_{n \rightarrow \infty} c_{2}(n) \leq \frac{e^{e^{c}}}{2}\left(1+\delta_{1}\right) .
$$

Proof. Using the notation introduced above we obtain from relation (3) that

$$
\sum_{k=0}^{f(n)} \sum_{1 \leq i_{1}<\cdots<i_{k} \leq T} \prod_{s=1}^{k} \prod_{j=1}^{n}\left(1-\frac{\ln n+x_{i_{s} j}}{n}\right) \geq \mu(n)-c_{2}(n) \cdot \frac{\ln ^{3} n}{n \cdot \ln \ln n},
$$

where

$$
c_{2}(n)=\frac{\mu(n)}{2}\left(1+\delta_{1}\right)\left(1+\frac{c}{\ln n}\right)^{2} \frac{1}{1-\frac{\ln n+c}{n}} .
$$

Taking into account inequalities (20) and passing to the limit as $n \rightarrow \infty$ we obtain

$$
\frac{\left(1+\delta_{1}\right)}{2}<\varliminf_{n \rightarrow \infty} c_{2}(n) \leq \varlimsup_{n \rightarrow \infty} c_{2}(n) \leq \frac{1}{2} e^{e^{c}}\left(1+\delta_{1}\right) .
$$

Lemma 3.5 is proved.

Lemma 3.6. Let conditions (2), (3), (6), and (19) hold. Then

$$
\begin{gathered}
\sum_{k: \frac{n}{2}\left(1-\frac{1}{\ln n}\right) \leq k \leq \frac{n}{2}\left(1+\frac{1}{\ln n}\right)}\left(\begin{array}{l}
n \\
k
\end{array}\right) \frac{1}{2^{n}} \sum_{l=f(n)}^{T}\left(\begin{array}{l}
T \\
l
\end{array}\right)\left(1-\frac{2(\ln n-c)}{n}\right)^{k l} \\
\leq\left(\frac{c_{3}(n)}{f(n)}\right)^{f(n)} \cdot \frac{1}{\sqrt{f(n)}} \cdot c_{4}(n)
\end{gathered}
$$

where $0<\underline{\lim }_{n \rightarrow \infty} c_{3}(n) \leq \varlimsup_{n \rightarrow \infty} c_{3}(n) \leq e^{2+c}$ and $\lim _{n \rightarrow \infty} c_{4}(n)=(2 \pi)^{-1 / 2}$.

Proof. For $(n / 2)(1-1 / \ln n) \leq k \leq(n / 2)(1+1 / \ln n)$, we have

$$
\left(1-\frac{2(\ln n-c)}{n}\right)^{k} \leq e^{-2 k(\ln n-c) / n} \leq \frac{1}{n} e^{c+1-c / \ln n} .
$$

Using the above estimate and applying the Stirling formula we obtain

$$
\begin{gathered}
\sum_{k: \frac{n}{2}\left(1-\frac{1}{\ln n}\right) \leq k \leq \frac{n}{2}\left(1+\frac{1}{\ln n}\right)}\left(\begin{array}{l}
n \\
k
\end{array}\right) \frac{1}{2^{n}} \sum_{l=f(n)}^{T}\left(\begin{array}{l}
T \\
l
\end{array}\right)\left(1-\frac{2(\ln n-c)}{n}\right)^{k l} \\
\leq\left(\frac{c_{3}(n)}{f(n)}\right)^{f(n)} \cdot \frac{1}{\sqrt{f(n)}} \cdot c_{4}(n)
\end{gathered}
$$


where

$$
\begin{gathered}
c_{3}(n)=\frac{T}{n} \cdot e^{2+c-c / \ln n}, \\
c_{4}(n)=\frac{1}{\sqrt{2 \pi}} \cdot\left(1-\frac{c_{3}(n)}{f(n)}\right)^{-1} .
\end{gathered}
$$

Considering (6) and (19) we get

$$
0<\varliminf_{n \rightarrow \infty} c_{3}(n) \leq \varlimsup_{n \rightarrow \infty} c_{3}(n) \leq e^{2+c}, \quad \lim _{n \rightarrow \infty} c_{4}(n)=(2 \pi)^{-1 / 2} .
$$

Lemma 3.6 is proved.

Lemma 3.7. Let conditions (2), (3), and (5) hold. Then

$$
\begin{aligned}
& \sum_{k=f(n)+1}^{T} \sum_{1 \leq i_{1}<\cdots<i_{k} \leq T} \frac{1}{2^{n}} \prod_{j=1}^{n}\left(1+\prod_{s=1}^{k}\left(1-\frac{2\left(\ln n+x_{i_{s} j}\right)}{n}\right)\right) \\
& \leq c_{5}(n) \cdot(\ln n)^{-\left(n \cdot c_{6}(n)\right) /(\ln n)^{q}} \\
& \quad+\left(\frac{c_{3}(n)}{f(n)}\right)^{f(n)} \cdot \frac{1}{\sqrt{f(n)}} \cdot c_{4}(n)+c_{7}(n) \cdot \exp \left\{-\frac{n}{2 \ln ^{2} n} c_{8}(n)\right\}
\end{aligned}
$$

where

$$
\begin{gathered}
c_{5}(n)=\frac{1}{\sqrt{2 \pi}}\left(1+\frac{(\ln n)^{q}}{n}\right) \\
c_{6}(n)=1-q-\frac{1}{\ln \ln n}\left(1-\ln \left(1-\frac{(\ln n)^{q}}{n}\right)+\frac{1}{2 n}(\ln n)^{1+q}-\frac{q(\ln n)^{q} \ln \ln n}{2 n}\right) \\
c_{7}(n)=\frac{1}{\sqrt{2 \pi}\left(1-\frac{1}{\ln ^{2} n}\right)}\left(\exp \left\{\frac{T}{n} e^{c+1-\frac{c}{\ln n}+2 \frac{\ln n-c}{n}}\right\}+\exp \left\{\frac{T}{n} e^{c-1+\frac{c}{\ln n}}\right\}\right), \\
c_{8}(n)=\left(1-\frac{1}{2 \ln n}-\frac{1}{2 \ln ^{2} n}-\frac{\ln ^{3} n}{n}\right) .
\end{gathered}
$$

If, additionally, condition (6) holds, then

$$
\begin{gathered}
\lim _{n \rightarrow \infty} c_{5}(n)=(2 \pi)^{-1 / 2}, \quad \lim _{n \rightarrow \infty} c_{6}(n)=1-q, \quad \lim _{n \rightarrow \infty} c_{8}(n)=1, \\
\sqrt{\frac{2}{\pi}}<\varliminf_{n \rightarrow \infty} c_{7}(n) \leq \varlimsup_{n \rightarrow \infty} c_{7}(n) \leq \frac{e^{c+1}+e^{c-1}}{\sqrt{2 \pi}} .
\end{gathered}
$$

Proof. The proof of Lemma 3.7 uses the result of Lemma 3.6 and follows the lines of the proof of Lemma 3.3.2 in [2].

Denote by $S(A)$ the maximal number of independent critical sets of a matrix $A$.

Lemma 3.8. Assume that conditions (2) and (3) hold for $k=0,1,2, \ldots, n$. Then

$$
\left|\mathrm{P}\{S(A)=k\}-\frac{e^{-\lambda} \cdot \lambda^{k}}{k !}\right| \leq 2 \mathrm{E} S_{1}(A)+Q(n, k),
$$

where $\mathrm{E} S_{1}(A)$ is found in Lemma 3.2, while $Q(n, k)$ is obtained in Lemma 3.1.

Proof. It follows from (13) that

$$
\begin{aligned}
\left|\mathrm{P}\{S(A)=k\}-\frac{e^{-\lambda} \cdot \lambda^{k}}{k !}\right| & \leq\left|\mathrm{P}\{S(A)=k\}-\mathrm{P}\left\{\xi_{n, T}=k\right\}\right|+Q(n, k) \\
& =\left|\mathrm{P}\left\{S_{1}(A)+\xi_{n, T}=k\right\}-\mathrm{P}\left\{\xi_{n, T}=k\right\}\right|+Q(n, k) .
\end{aligned}
$$


Applying the following relations

$$
\begin{gathered}
\mathrm{P}\left\{S_{1}(A)+\xi_{n, T}=k\right\}=\sum_{l=0}^{k} \mathrm{P}\left\{S_{1}(A)=l, \xi_{n, T}=k-l\right\}, \\
\left|\mathrm{P}\left\{S_{1}(A)=0, \xi_{n, T}=k\right\}-\mathrm{P}\left\{\xi_{n, T}=k\right\}\right| \leq \mathrm{P}\left\{S_{1}(A) \geq 1\right\}
\end{gathered}
$$

and Chebyshev's inequality we prove that

$$
\left|\mathrm{P}\{S(A)=k\}-\frac{e^{-\lambda} \cdot \lambda^{k}}{k !}\right| \leq 2 \mathrm{P}\left\{S_{1}(A) \geq 1\right\}+Q(n, k) \leq 2 \mathrm{E} S_{1}(A)+Q(n, k) .
$$

Lemma 3.8 is proved.

\section{Proof of Theorem 2.1}

First we obtain a bound for $\mathrm{E} S_{1}(A)$. For $k \geq 0$, put

$$
\begin{gathered}
\Delta_{1}(k)=\sum_{1 \leq i_{1}<\cdots<i_{k} \leq T} \frac{1}{2^{n}} \prod_{j=1}^{n}\left(1+\prod_{s=1}^{k}\left(1-\frac{2\left(\ln n+x_{i_{s} j}\right)}{n}\right)\right), \\
\Delta_{2}(k)=\sum_{1 \leq i_{1}<\cdots<i_{k} \leq T} \prod_{s=1}^{k} \prod_{j=1}^{n}\left(1-\frac{\ln n+x_{i_{s} j}}{n}\right) .
\end{gathered}
$$

Lemma 3.2 implies that

$$
\mathrm{E} S_{1}(A) \leq \sum_{k=0}^{f(n)} \Delta_{1}(k)+\sum_{k=f(n)+1}^{T} \Delta_{1}(k)-\sum_{k=0}^{f(n)} \Delta_{2}(k) .
$$

By Lemmas 3.4 and 3.5 we get

$$
\sum_{k=0}^{f(n)} \Delta_{1}(k)-\sum_{k=0}^{f(n)} \Delta_{2}(k) \leq \mu(n)(1+\delta) \Gamma(n)+c_{2}(n) \frac{\ln ^{3} n}{n \cdot \ln \ln n} .
$$

Combining (36) and the estimate for the sum $\sum_{k=f(n)+1}^{T} \Delta_{1}(k)$ obtained in Lemma 3.7 we derive the inequality

$$
\mathrm{E} S_{1}(A) \leq \mu(n)(1+\delta) \Gamma(n)+F(n)
$$

where

$$
\begin{aligned}
F(n)= & c_{2}(n) \frac{\ln ^{3} n}{n \cdot \ln \ln n}+c_{5}(n) \cdot(\ln n)^{-n \cdot c_{6}(n) /(\ln n)^{q}} \\
& +\left(\frac{c_{3}(n)}{f(n)}\right)^{f(n)} \cdot \frac{1}{\sqrt{f(n)}} \cdot c_{4}(n)+c_{7}(n) \cdot \exp \left\{-\frac{n}{2 \ln ^{2} n} c_{8}(n)\right\}
\end{aligned}
$$

and where the coefficients $c_{2}(n), c_{3}(n), \ldots, c_{8}(n)$ are defined by equalities (29)-(35), respectively.

According to (37) and Lemma 3.8 ,

$$
\left|\mathrm{P}\{S(A)=k\}-\frac{e^{-\lambda} \cdot \lambda^{k}}{k !}\right| \leq 2\langle\mu(n)(1+\delta) \Gamma(n)+F(n)\rangle+Q(n, k) .
$$

Considering the explicit expression for $\Gamma(n)$ we write

$$
\left|\mathrm{P}\{S(A)=k\}-\frac{e^{-\lambda} \cdot \lambda^{k}}{k !}\right| \leq 2(1+\delta) c_{11}(n) u+2 F(n)+Q(n, k),
$$


where

$$
c_{11}(n)=\mu(n)+\mu(n) \frac{u}{2}(1+\delta) \exp \{u(1+\delta)\} .
$$

The latter inequality implies

$$
\left|\mathrm{P}\{r(A)=T-k\}-\frac{e^{-\lambda} \cdot \lambda^{k}}{k !}\right| \leq 2(1+\delta) c(n, k) \frac{\ln ^{4} n}{n(\ln \ln n)^{2}},
$$

since $r(A)+S(A)=T$ (the latter equality is proved in [2]), where

$$
c(n, k)=c_{11}(n)+\frac{F(n)}{(1+\delta) u}+\frac{Q(n, k)}{2 u(1+\delta)}
$$

and $c_{11}(n), F(n)$, and $Q(n, k)$ are defined by equalities (39), (38), and (14), respectively.

Theorem 2.1 is proved.

\section{Proof of Theorem 2.2}

Let $A$ be the matrix defined in Theorem 2.2 and let $A^{\prime}$ denote the transpose matrix of $A$. Theorem 2.1 can be applied to $A^{\prime}$, since the analogue of condition (5) written for $A^{\prime}$ is

$$
\frac{n}{T} \leq 1-v+\frac{v^{2}}{1-v}
$$

where

$$
v=\frac{\log _{2} \ln T}{(\ln T)^{q}} .
$$

Note that the above condition follows from $T / n \geq 1+v$.

The term $v^{2}(1-v)^{-1}$ on the right hand side of (41) changes the coefficient $c_{6}(n)$ in Theorem 2.2. The changed coefficient is equal to

$$
c_{6}(T)+\frac{v}{1-v},
$$

where $c_{6}(n)$ is defined by equality (33).

Theorem 2.2 is proved.

\section{Proof of Theorem 2.3}

Denote by $\mu_{n, T}$ the number of solutions of system (11). The probability $P_{n, T}$ that system (1) is consistent equals the probability that the system has at least one solution, that is, $P_{n, T}=\mathrm{P}\left\{\mu_{n, T}>0\right\}$.

We find an explicit expression for the probability of the event $\left\{\mu_{n, T}>0\right\}$ given that the rank $r(A)$ of the matrix $A$ is equal to $r$. Without loss of generality we assume that the rows $1,2, \ldots, r$ are linearly independent in the matrix $A$. Then each of the rows $r+1, \ldots, T$ is a linear combination of the first $r$ rows. In order that the system be consistent, its right hand sides $b_{r+1}, \ldots, b_{T}$ should be such that

$$
\varepsilon_{1 i}^{*} b_{1} \oplus \cdots \oplus \varepsilon_{r i}^{*} b_{r}=b_{i}, \quad i=r+1, \ldots, T,
$$

where $\varepsilon_{1 i}^{*}, \ldots, \varepsilon_{r i}^{*}$ assume only the values 0 or 1 .

The probability of an arbitrary relation among (42) is equal to

$$
\mathrm{P}\left\{b_{\nu_{1}} \oplus \cdots \oplus b_{\nu_{s}}=b_{i}\right\}=\frac{1}{2}\left(1+\left(\prod_{j=1}^{s(i)} \varepsilon_{\nu_{j}}(n)\right) \varepsilon_{i}(n)\right),
$$

where $1 \leq s \leq r, s=s(i), i=r+1, \ldots, T$, and $s(i)$ is the number of nonzero elements among $\varepsilon_{1 i}^{*}, \ldots, \varepsilon_{r i}^{*}$ on the left hand side of (42). Equality (43) can be checked by induction. 
Taking into account (9), (10), (43), and

$$
P_{n, T}=\sum_{k=0}^{T} \mathrm{P}\{r(A)=T-k\} \cdot \mathrm{P}\left\{\mu_{n, T}>0 / r(A)=T-k\right\}
$$

we obtain

$$
\begin{aligned}
\sum_{k=0}^{T} \mathrm{P} & \{r(A)=T-k\}\left(\frac{1-\tilde{\varepsilon}(n)}{2}\right)^{k} \\
\leq P_{n, T} & \leq \sum_{k=0}^{T} \mathrm{P}\{r(A)=T-k\}\left(\frac{1+\tilde{\varepsilon}(n)}{2}\right)^{k} .
\end{aligned}
$$

Further we show that

$$
P_{n, T}-e^{-\lambda / 2} \leq \alpha_{1} f(n)+\tilde{\varepsilon}(n) .
$$

First we estimate from above the difference $P_{n, T}-e^{-\lambda / 2}$ by using the right hand side of relation (44):

$$
P_{n, T}-e^{-\lambda / 2} \leq \sum_{k=0}^{T}\left(\mathrm{P}\{r(A)=T-k\}-\frac{e^{-\lambda} \lambda^{k}}{k !}\right) \frac{1}{2^{k}}+\sum_{k=0}^{T} \frac{e^{-\lambda} \lambda^{k}}{k !} \frac{1}{2^{k}}-e^{-\lambda / 2}+\theta,
$$

where

$$
\theta=\sum_{k=0}^{T}\left(\mathrm{P}\{r(A)=T-k\}-\frac{e^{-\lambda} \lambda^{k}}{k !}\right) \frac{(1+\tilde{\varepsilon}(n))^{k}-1}{2^{k}}+\sum_{k=0}^{T} \frac{e^{-\lambda} \lambda^{k}}{k !} \frac{(1+\tilde{\varepsilon}(n))^{k}-1}{2^{k}} .
$$

Now we use Theorem 2.1 and get

$$
P_{n, T}-e^{-\lambda / 2} \leq f(n)+\theta .
$$

We again use Theorem 2.1 to estimate the sum $\theta$ from above:

$$
\theta \leq \frac{f(n)}{2} \sum_{k=0}^{T} \frac{(1+\tilde{\varepsilon}(n))^{k}}{2^{k}}+e^{-\lambda} \sum_{k=0}^{T} \frac{\lambda^{k}}{k !} \frac{\tilde{\varepsilon}(n) 2^{k}}{2^{k}},
$$

whence

$$
\theta \leq \frac{f(n)}{2} \frac{1}{1-(1+\tilde{\varepsilon}(n)) / 2}+\tilde{\varepsilon}(n) .
$$

Bounds (46) and (47) imply (45).

Now we show that

$$
P_{n, T}-e^{-\lambda / 2} \geq-\alpha_{2} f(n)-\tilde{\varepsilon}(n) .
$$

Indeed, using the left hand side of relation (44) we estimate the difference $P_{n, T}-e^{-\lambda / 2}$ from below:

$$
P_{n, T}-e^{-\lambda / 2} \geq \sum_{k=0}^{T}\left(\mathrm{P}\{r(A)=T-k\}-\frac{e^{-\lambda} \lambda^{k}}{k !}\right) \frac{1}{2^{k}}+\sum_{k=0}^{T} \frac{e^{-\lambda} \lambda^{k}}{k !} \frac{1}{2^{k}}-e^{-\lambda / 2}+\theta,
$$

where

$$
\theta=\sum_{k=0}^{T}\left(\mathrm{P}\{r(A)=T-k\}-\frac{e^{-\lambda} \lambda^{k}}{k !}\right) \frac{(1-\tilde{\varepsilon}(n))^{k}-1}{2^{k}}+\sum_{k=0}^{T} \frac{e^{-\lambda} \lambda^{k}}{k !} \frac{(1-\tilde{\varepsilon}(n))^{k}-1}{2^{k}} .
$$

Theorem 2.1 implies that

$$
P_{n, T}-e^{-\lambda / 2} \geq-f(n)+\sum_{k=0}^{T} \frac{e^{-\lambda} \lambda^{k}}{k !} \frac{1}{2^{k}}-e^{-\lambda / 2}+\theta,
$$


whence

$P_{n, T}-e^{-\lambda / 2} \geq-f(n)-\sum_{k=T+1}^{\infty} \frac{e^{-\lambda}(\lambda / 2)^{k}}{k !}+\theta \geq-f(n)-e^{-\lambda} \sum_{k=T+1}^{\infty}\left(\frac{\lambda e}{2 k}\right)^{k} \frac{1}{\sqrt{2 \pi k}}+\theta$.

It follows from (3) and (4) that the inequality $\lambda e /(2 T)<1$ holds for $n>2^{-1} e^{1+c}$. For this $n$, we get

$$
P_{n, T}-e^{-\lambda / 2} \geq-f(n)(1+\Delta(n, T))+\theta,
$$

where $\Delta(n, T)$ satisfies the bounds (12).

The sum $\theta$ is estimated from below with the help of Theorem 2.1

$$
\theta \geq-\frac{f(n)}{2} \sum_{k=0}^{T} \frac{(1-\tilde{\varepsilon}(n))^{k}}{2^{k}}-e^{-\lambda} \sum_{k=0}^{T} \frac{\lambda^{k}}{k !} \frac{\tilde{\varepsilon}(n) 2^{k}}{2^{k}},
$$

whence

$$
\theta \geq-\frac{f(n)}{2} \frac{1}{1-\frac{1-\tilde{\varepsilon}(n)}{2}}-\tilde{\varepsilon}(n)
$$

Relation (49) together with (12) and (50) implies (48).

The upper and lower bounds (45) and (48) prove relation (11).

Theorem 2.3 is proved.

\section{The Limit Distribution of the RANK of a SPARSE Boolean RANDOM Matrix}

Theorem 7.1. I. Let the assumptions of Theorem 2.1 hold. If

$$
\lambda \rightarrow \omega, \quad n \rightarrow \infty,
$$

and $0<\omega<\infty$, then

$$
\mathrm{P}\{r(A)=T-k\} \rightarrow e^{-\omega} \frac{\omega^{k}}{k !}, \quad n \rightarrow \infty,
$$

for a fixed $k=0,1,2, \ldots$.

II. Let conditions (2), (3), (5), and (51) hold. If $\omega>0$, then $\omega<\infty$ and relation (52) is valid.

The proof of Theorem 7.1 is easy in view of Theorem 2.1

Corollary 7.1. Let condition (2) hold for

$$
x_{i j}=x, \quad i=1, \ldots, T, j=1, \ldots, n,
$$

where $x$ is a fixed number. If $T / n \rightarrow \alpha$ as $n \rightarrow \infty$ for some $0<\alpha<1$, then relation (52) is valid for $\omega=\alpha e^{-x}$.

Theorem 7.2. I. Let the assumptions of Theorem 2.2 hold. If

$$
\lambda_{1} \rightarrow \omega_{1}, \quad n \rightarrow \infty,
$$

and $0<\omega_{1}<\infty$, then

$$
\mathrm{P}\{r(A)=n-k\} \rightarrow e^{-\omega_{1}} \frac{\omega_{1}^{k}}{k !}, \quad n \rightarrow \infty,
$$

for a fixed $k=0,1,2, \ldots$.

II. Let conditions (3), (7), and (53) hold. If

$$
1+\frac{\log _{2} \ln T}{(\ln T)^{q}} \leq \frac{T}{n}
$$

$q=$ const, $0<q<1$, and $\omega_{1}>0$, then $\omega_{1}<\infty$ and relation (54) is valid. 
The proof of Theorem 7.2 is easy in view of Theorem 2.2

Corollary 7.2. Let condition (2) hold for

$$
x_{i j}=x, \quad i=1, \ldots, T, j=1, \ldots, n,
$$

where $x$ is a fixed number. If $T / n \rightarrow \alpha$ as $n \rightarrow \infty$ for some $1<\alpha<\infty$, then relation (54) is valid for $\omega_{1}=\alpha^{-1} e^{-x}$.

The proof of Corollary 7.1 (Corollary 17.2) follows from the observation that the assumptions of the first (second) statement of Theorem 7.1 (Theorem [7.2) obviously hold under the assumptions of the corresponding corollary.

Remark 7.1. Corollaries 7.1 and 7.2 are proved in [2, Theorem 3.3.1] and [2, Theorem 3.3.2], respectively.

\section{The asymptotic Behavior of the probability $P_{n, T}$}

Theorem 8.1. Let conditions (22), (3), (5), (6), and (8) hold. If

$$
\lambda \rightarrow \lambda^{*}
$$

and

$$
\tilde{\varepsilon}(n) \rightarrow 0
$$

as $n \rightarrow \infty$, then

$$
0<\lambda^{*}<\infty
$$

and moreover

$$
P_{n, T} \rightarrow \exp \left\{-\frac{\lambda^{*}}{2}\right\}, \quad n \rightarrow \infty .
$$

Inequality (57) can easily be derived from (3), (5), and (6). Relation (58) follows from Theorem 2.3 by (55) and (56).

Corollary 8.1. Assume that condition (2) is valid for

$$
x_{i j}=x, \quad i=1, \ldots, T, j=1, \ldots, n,
$$

where $x$ is a fixed number and $T / n \rightarrow \alpha$ as $n \rightarrow \infty$ for some $0<\alpha<1$. Then relation (58) holds for $\lambda^{*}=\alpha e^{-x}$.

\section{BIBLIOGRAPHY}

1. G. V. Balakin, The distribution of the rank of random matrices over a finite field, Teor. Verojatnost. i Primenen. XIII (1968), no. 4, 631-641; English transl. in Theor. Probab. Appl. 13 (1968), no. 4, 594-605. MR0243571 (39:4892)

2. V. F. Kolchin, Random Graphs, Fizmatlit, Moscow, 2000, 256 pp. (Russian) MR.1812261 (2002e:60014)

3. V. I. Masol, Moments of the number of solutions of a system of random Boolean equations, Random Oper. Stochastic Equations 1 (1993), no. 2, 171-179. MR1254185 (94h:60089) 
4. V. I. Masol, Invariance theorems for systems of random Boolean equations, Sixth Intern. Vilnius Conf. of Probability Theory and Math. Statist., Abstracts of Communications, 1993, pp. 19-20.

5. B. A. Sevast'yanov, A Course in Probability Theory and Mathematical Statistics, "Nauka", Moscow, 1982. (Russian) MR712294(85a:60006)

Department of Probability Theory and Mathematical Statistics, Faculty for Mechanics and Mathematics, National Taras Shevchenko University, Academician Glushkov Avenue 6, KYIV 03127, UKRAINE

E-mail address: vimasol@ukr.net

Department of Probability Theory and Mathematical Statistics, Faculty for Mechanics and Mathematics, National Taras Shevchenko University, Academician Glushkov Avenue 6 , KYIV 03127, UKRAINE

E-mail address: Popereshnyak_sv@mail.ru

Received 27/DEC/2005

Translated by N. SEMENOV 